\title{
SOME ELLIPTIC PROBLEMS WITH NONLINEAR BOUNDARY CONDITIONS
}

\author{
C. MORALES-RODRIGO AND A. SUÁREZ* \\ Dpto. Ecuaciones Diferenciales y Análisis Numérico, \\ C/ Tarfia s/n, 41080, \\ Univ. of Sevilla, Seville, Spain \\ E-mails: cristianmatematicas@yahoo.com and suarez@us.es
}

\author{
In memory of Prof. J. Esquinas
}

\begin{abstract}
This paper concerns with some elliptic equations with non-linear boundary conditions. Sub-supersolution and bifurcation methods are used in order to obtain existence, uniqueness or multiplicity of positive solutions.
\end{abstract}

\section{Introduction}

In this paper we study positive solutions of some nonlinear elliptic problems with mixed nonlinear boundary conditions. Throughout it, we consider the following assumptions:

(1) $\Omega \subset \mathbb{R}^{N}, N \geq 1$, is a bounded domain with boundary $\partial \Omega$ of class $\mathcal{C}^{2}$. Moreover,

$$
\partial \Omega:=\Gamma_{0} \cup \Gamma_{1},
$$

where $\Gamma_{0}$ and $\Gamma_{1}$ denote two disjoint open and closed sets in the relative topology of $\partial \Omega$.

(2) $L$ is a uniformly elliptic differential operator in $\Omega$ of the form

$$
L:=-\sum_{i, j=1}^{N} a_{i j} \frac{\partial^{2}}{\partial x_{i} \partial x_{j}}+\sum_{i=1}^{N} b_{i} \frac{\partial}{\partial x_{i}}+c,
$$

with coefficients $a_{i j}=a_{j i} \in \mathcal{C}^{2, \alpha}(\bar{\Omega}), b_{i} \in \mathcal{C}^{1, \alpha}(\bar{\Omega})$ and $c \in \mathcal{C}^{\alpha}(\bar{\Omega})$, $\alpha \in(0,1)$.

*Supported by the Spanish Ministry of Science and Technology under grant BFM200306446. 
(3) We define the mixed boundary operator, $B$, by

$$
B u:=\left\{\begin{array}{l}
u \text { on } \Gamma_{0}, \\
\mathcal{B} u \text { on } \Gamma_{1},
\end{array}\right.
$$

where the operator $\mathcal{B}:=\partial_{\nu}+b$ with $\nu \in \mathcal{C}^{1}\left(\Gamma_{1}, \mathbb{R}^{N}\right)$ an outward pointing nowhere tangent vector-field and $b \in \mathcal{C}^{1, \alpha}\left(\Gamma_{1}\right)$.

In this paper we study the following problems where $a$ is a positive or negative regular function on $\Gamma_{1}$ and $0<q<1<p, r$. We first study an elliptic equation with a logistic term on the boundary

$$
\begin{cases}L u=0 & \text { in } \Omega, \\ u=0 & \text { on } \Gamma_{0}, \\ \mathcal{B} u=\mu u+a(x) u^{r} & \text { on } \Gamma_{1},\end{cases}
$$

where $\mu \in \mathbb{R}$ will be regarded as bifurcation parameter. We do not know previous works in which (3) was analyzed. We characterize the existence, uniqueness and stability of positive solution in terms of the parameter $\mu$ (see Theorem 5.2).

Second, we study of the sublinear-superlinear equation

$$
\begin{cases}-\Delta u=\lambda u-u^{p} & \text { in } \Omega, \\ \frac{\partial u}{\partial n}=u^{r} & \text { on } \partial \Omega,\end{cases}
$$

where $n$ is the outward normal vector-field of $\Omega$. (The case $-u^{r}$ instead $u^{r}$ has been studied in Ref. ${ }^{8}$.) Equation (4) has attracted a lot of attention in the last years with $\lambda=0$, see Refs. ${ }^{6},{ }^{10},{ }^{18},{ }^{21},{ }^{22}$ and ${ }^{26}$, among others, where basically the equation and its corresponding parabolic problem were analyzed in the particular case $\lambda=0$, and in Refs. ${ }^{28},{ }^{29}$ where the local bifurcation was studied. We complete this study giving existence, nonexistence and stability results in function of $\lambda$ (see Theorem 5.3).

Finally, we study the concave-convex equation

$$
\left\{\begin{array}{l}
L u=\lambda m(x) u^{q} \text { en } \Omega, \\
\frac{\partial u}{\partial n}=a(x) u^{r} \quad \text { en } \partial \Omega .
\end{array}\right.
$$

where $m \in \mathcal{C}(\bar{\Omega})$ is nonnegative and non-trivial. (5) was studied previously in Ref. ${ }^{14}$ when $L u=-\Delta u+u$, and $m \equiv a \equiv 1$ by variational methods. When $a<0$ we prove that there exists a positive solution of (5) if and only if $\lambda>0$. If $a>0$ we complete and improve the results of Ref. ${ }^{14}$ (see Theorems 5.4 and 5.5). 
In order to study these equations we employ mainly sub-supersolution and bifurcation methods. We present in Sect. 2 results related with principal eigenvalues associated to these problems. In Sec. 3 we prove a general result of bifurcation from the trivial solution when the bifurcation parameter appears in both equation and boundary. As consequence, we can use it for equations (3) and (4). For the study of (5) we need a different result of bifurcation, where the parameter is in front of a non-linear term. In Sec. 4 we present results concerning to uniqueness, stability and a-priori bounds of positive solutions for general equations with nonlinear boundary conditions. Finally, in Sec. 5 we apply the results to the cited equations.

\section{Some Preliminaries Results: eigenvalues problems}

Along this paper, we use the positive cone

$$
P:=\left\{u \in \mathcal{C}^{1}(\bar{\Omega}): u \geq 0, u \neq 0 \text { in } \Omega \cup \Gamma_{1}, B u=0 \text { on } \partial \Omega\right\},
$$

and we say that $u$ is positive if $u \in P$ and that $u$ is strongly positive if $u \in \operatorname{int}(P):=\left\{u \in P: u>0\right.$ in $\Omega \cup \Gamma_{1}, \partial u / \partial n<0$ on $\left.\Gamma_{0}\right\}$, where $n$ is the outward normal vector-field of $\Omega$. On the other hand, the mixed operator $B+m, m \in \mathcal{C}\left(\Gamma_{1}\right)$, means a similar operator to (2) with $b+m$ instead of $b$ in $\mathcal{B}$. Finally, given two functions $u, v$ we write $(u, v)>0$ if $u, v \geq 0$ and some of the inequalities non-trivial.

Consider the eigenvalue problem

$$
\left\{\begin{array}{l}
L \varphi=\lambda \varphi \text { in } \Omega \\
B \varphi=0 \text { on } \partial \Omega .
\end{array}\right.
$$

H. Amann ${ }^{2}$ proved the existence of a unique simple eigenvalue, the principal eigenvalue, whose associated eigenfunction can be chosen strongly positive in $\Omega$. We denote this eigenvalue by $\sigma_{1}[L, B] . \sigma_{1}[L, D]$ and $\sigma_{1}[L, \mathcal{N}]$ stand for the principal eigenvalues under Dirichlet and Neumann homogeneous boundary conditions, respectively.

Some properties of $\sigma_{1}[L, B]$ have been studied in details by S. CanoCasanova and J. López-Gómez ${ }^{9}$ (see also Ref. ${ }^{4}$ ), we state some of them.

\section{Proposition 2.1.}

(1) $\sigma_{1}[L, B]>0$ if and only if there exists a positive supersolution of $(L, B, \Omega)$, i.e., a positive function $\bar{u}$ such that $L \bar{u} \geq 0$ in $\Omega$ and $B \bar{u} \geq 0$ on $\partial \Omega$ with some inequality strict.

(2) The map $q \in L^{\infty}(\Omega) \mapsto \sigma_{1}[L+q, B]$ is increasing and continuous. 
(3) The map $m \in \mathcal{C}\left(\Gamma_{1}\right) \mapsto \sigma_{1}[L, B+m]$ is increasing and continuous.

(4) Suppose $\Gamma_{1} \neq \emptyset$ and consider a sequence $b_{n} \in \mathcal{C}\left(\Gamma_{1}\right)$ such that $\lim _{n \rightarrow+\infty} \min _{\Gamma_{1}} b_{n}=+\infty$. Then,

$$
\lim _{n \rightarrow+\infty} \sigma_{1}\left[L, B+b_{n}\right]=\sigma_{1}[L, D] .
$$

(5) Suppose $\Gamma_{1} \neq \emptyset$, then $\sigma_{1}[L, B]<\sigma_{1}[L, D]$.

Consider now the eigenvalue problem

$$
\begin{cases}L \varphi=\lambda m(x) \varphi & \text { in } \Omega, \\ \varphi=0 & \text { on } \Gamma_{0} \\ \mathcal{B} \varphi=\lambda r(x) \varphi & \text { on } \Gamma_{1} .\end{cases}
$$

We suppose the following condition

$$
m \in \mathcal{C}^{\alpha}(\bar{\Omega}), \quad r \in \mathcal{C}^{1, \alpha}\left(\Gamma_{1}\right), \exists \mu \geq 0 \text { such that }(c+\mu m, b+\mu r)>0 .
$$

The following result provides us the existence of principal eigenvalue of (6). The second paragraph gives a characterization of the principal eigenvalue of (6) when $m \equiv 0$, i.e., an eigenvalue problem at the boundary, the classical Steklov problem. In our acknowledge this result is new, although it nearly follows by the results on Ref. ${ }^{9}$ (see Ref. ${ }^{15}$ where a particular result is obtained.)

Theorem 2.1. Assume $(m, r)>0$. Then:

(1) Under condition (7), the eigenvalue problem (6) has a unique principal eigenvalue, $\gamma_{1}[L, B]$, it is simple and its associated eigenfunction can be chosen strongly positive in $\Omega$.

(2) If $m \equiv 0$ and $r>0$, then, the principal eigenvalue exists for (6), denoted by $\lambda_{1}[L, B]$, if and only if $\sigma_{1}[L, D]>0$. Moreover, its associated eigenfunction can be chosen strongly positive in $\Omega$.

Proof: The first paragraph follows with the same kind of arguments used in Theorem 2.2 of $\mathrm{H}$. Amann ${ }^{3}$ where $\Gamma_{0}=\emptyset$.

It is clear that $\lambda_{1}$ is a principal eigenvalue of (6) with $m \equiv 0$ if and only if $\mu\left(\lambda_{1}\right)=0$ where $\mu(\lambda):=\sigma_{1}[L, B-\lambda r(x)]$.

We know by Proposition 2.1 that $\lim _{\lambda \rightarrow-\infty} \mu(\lambda)=\sigma_{1}[L, D], \mu(\lambda)$ is a decreasing and continuous function. So, it suffices to prove that $\lim _{\lambda \rightarrow+\infty} \mu(\lambda)=-\infty$. Suppose the contrary, then $\lim _{\lambda \rightarrow+\infty} \mu(\lambda)=-l$. Take $k \in \mathbb{R}$ large enough such that $k+c(x)>0$ and $k>l$ then, first part 
of the Theorem can be applied to the eigenvalue problem

$$
\begin{cases}L \varphi+k \varphi=\widetilde{\mu} \varphi & \text { in } \Omega \\ \varphi=0 & \text { on } \Gamma_{0} \\ \mathcal{B} \varphi=\lambda r(x) \varphi & \text { on } \Gamma_{1}\end{cases}
$$

Hence, there is a principal eigenvalue $\widetilde{\lambda}_{1}$ that verifies $0=\widetilde{\mu}\left(\widetilde{\lambda}_{1}\right)=\mu\left(\widetilde{\lambda}_{1}\right)+k$. This is a contradiction.

The following result will be very useful along this work.

Lemma 2.1. Assume (7) and $(m, r)>0$. Then $\gamma_{1}[L, B]>0 \Longleftrightarrow$ $\sigma_{1}[L, B]>0$

Proof: We know by Theorem 2.1 that $\gamma_{1}[L, B]$ exists, and it is the unique zero of the application

$$
\mu(\sigma)=\sigma_{1}[L-\sigma m, B-\sigma r] .
$$

Since $\mu$ is a decreasing function, then $\mu(0)>0$ implies $\mu\left(\sigma_{0}\right)=0$ for $\sigma_{0}=\gamma_{1}[L, B]>0$ and the contrary.

\section{Bifurcation Results for Equations with Nonlinear Boundary}

Consider the nonlinear equation

$$
\begin{cases}L u=\lambda m(x) u+f(x, u) & \text { in } \Omega, \\ u=0 & \text { on } \Gamma_{0}, \\ \mathcal{B} u=\lambda r(x) u+g(x, u) & \text { on } \Gamma_{1},\end{cases}
$$

where $f \in \mathcal{C}^{\alpha}(\bar{\Omega} \times \mathbb{R}), g \in \mathcal{C}^{1, \alpha}\left(\Gamma_{1} \times \mathbb{R}\right)$, such that

$$
f(x, 0)=0 \forall x \in \Omega, \quad g(x, 0)=0 \forall x \in \Gamma_{1},
$$

$(m, r)>0$ and satisfy condition (7) and $\lambda$ is a bifurcation parameter.

Remark 3.1. Due to the condition (7) we can assume, adding $\mu m$ and $\mu r$ to both sides of $(8)$, that $(c, b)>0$.

Now, we reduce the equation (8) to a suitable equation for compact operators. Define $\mathcal{C}_{\Gamma_{0}}^{\alpha}(\bar{\Omega})=\left\{v \in \mathcal{C}^{\alpha}(\bar{\Omega}): v_{\mid \Gamma_{0}}=0\right\}$ (analogously it can defined $\left.\mathcal{C}_{\Gamma_{0}}^{2, \alpha}(\bar{\Omega})\right)$ and the map $K_{1}: \mathcal{C}_{\Gamma_{0}}^{\alpha}(\bar{\Omega}) \rightarrow \mathcal{C}_{\Gamma_{0}}^{2, \alpha}(\bar{\Omega})$ by, given $f, K_{1}(f)=u$ where $u$ is the unique solution of the problem

$$
\left\{\begin{array}{l}
L u=f \text { in } \Omega \\
B u=0 \text { on } \partial \Omega .
\end{array}\right.
$$


We can extend this operator to $\mathcal{C}_{\Gamma_{0}}(\bar{\Omega})$. Thanks to elliptic regularity results, this new operator, denoted again by $K_{1}$, is compact as operator from $\mathcal{C}_{\Gamma_{0}}(\bar{\Omega})$ to $\mathcal{C}_{\Gamma_{0}}(\bar{\Omega})$. We define now $K_{2}: \mathcal{C}\left(\Gamma_{1}\right) \rightarrow \mathcal{C}_{\Gamma_{0}}^{2, \alpha}(\bar{\Omega})$ by, given $g, K_{2}(g)=u$ with $u$ the unique solution of the problem

$$
\left\{\begin{array}{l}
L u=0 \text { in } \Omega \\
u=0 \text { on } \Gamma_{0} \\
\mathcal{B} u=g \text { on } \Gamma_{1} .
\end{array}\right.
$$

Again, it can be proved that the operator $K_{2}: \mathcal{C}_{\Gamma_{0}}(\partial \Omega) \rightarrow \mathcal{C}_{\Gamma_{0}}(\bar{\Omega})$ is compact. Denote by $\gamma: \mathcal{C}(\bar{\Omega}) \rightarrow \mathcal{C}\left(\Gamma_{1}\right)$ the trace operator. Following the same kind of arguments that in Ref. ${ }^{3}$, Lemma 4.1, and denoting $M, R, F$ and $G$ by the Nemitski operators associated to $m(x) u, r(x) u, f$ and $g$ respectively, we have

Proposition 3.1. $u$ satisfies $u=K_{1}[\lambda M(u)+F(u)]+K_{2}[\lambda R(\gamma(u))+$ $G(\gamma(u))]$ if and only if $u$ is a classical solution of (8).

Since we are only interested in non-negative solutions of (8), we rewrite (8) as a problem with only non-negative solutions. Let $u^{+}=\max \{u, 0\}$.

Lemma 3.1. If $u$ is a solution of

$$
\begin{cases}L u=\lambda m(x) u^{+}+f\left(x, u^{+}\right) & \text {in } \Omega \\ u=0 & \text { on } \Gamma_{0} \\ \mathcal{B} u=\lambda r(x) u^{+}+g\left(x, u^{+}\right) & \text {on } \Gamma_{1}\end{cases}
$$

then $u \geq 0$.

Proof: Suppose that the problem (10) possesses solution $u$ such that there exists a connected component $\Omega_{1} \subset \Omega$ of the set $\Omega^{\prime}=\{x \in \Omega: u(x)<0\}$ such that $u<0$ in $\Omega_{1}$. Observe that $\partial \Omega_{1} \cap \Gamma_{1} \neq \emptyset$. Indeed, if $\Omega_{1} \subset \Omega$, then

$$
L u=0 \quad \text { in } \Omega_{1}, \quad u=0 \quad \text { on } \partial \Omega_{1} .
$$

Since $c \geq 0$, then by the maximum principle $u \equiv 0$ in $\Omega_{1}$. Hence, $\partial \Omega_{1} \cap \Gamma_{1} \neq$ $\emptyset$. Due to $L u \geq 0$ in $\Omega_{1}$ and $c \geq 0$ then, by the maximum principle, the minimum of $u$ must be attained on $\partial \Omega_{1}$. As $u<0$ in $\Omega_{1}$ and $u=0$ in $\partial \Omega_{1} \cap \Gamma_{0}$ then, minimum must be attained on $\partial \Omega_{1} \cap \Gamma_{1}$, but in such points we have

$$
\frac{\partial u}{\partial \nu}=-b(x) u \geq 0
$$

contradicting Hopf's Lemma (see Lemma 3.4 in Ref. ${ }^{16}$ ).

Remark 3.2. Lemma 3.1 is still true if $f(x, 0) \geq 0$ and $g(x, 0) \geq 0$. 
Consider the maps $\Phi_{\lambda}, \Phi_{\lambda}^{t}: \mathcal{C}_{\Gamma_{0}}(\bar{\Omega}) \rightarrow \mathcal{C}_{\Gamma_{0}}(\bar{\Omega})$ defined by

$$
\begin{aligned}
& \Phi_{\lambda}(u)=u-K_{1}\left[\lambda M\left(u^{+}\right)+F\left(u^{+}\right)\right]-K_{2}\left[\lambda R\left(\gamma\left(u^{+}\right)\right)+G\left(\gamma\left(u^{+}\right)\right)\right], \\
& \Phi_{\lambda}^{t}(u)=u-t K_{1}\left[\lambda M\left(u^{+}\right)+F\left(u^{+}\right)\right]-t K_{2}\left[\lambda R\left(\gamma\left(u^{+}\right)\right)+G\left(\gamma\left(u^{+}\right)\right)\right], t \geq 0 .
\end{aligned}
$$

Thanks to Proposition 3.1 and Lemma 3.1, $u$ is a classical nonnegative solution of (8) if and only if $\Phi_{\lambda}(u)=0$ in $\mathcal{C}_{\Gamma_{0}}(\bar{\Omega})$.

Assume that

$$
\lim _{s \rightarrow 0^{+}} \frac{f(x, s)}{s}=0 \text { unif. in } \bar{\Omega}, \quad \lim _{s \rightarrow 0^{+}} \frac{g(x, s)}{s}=0 \text { unif. on } \Gamma_{1} .
$$

Finally, denote by $\gamma_{1}:=\gamma_{1}[L, B]$, and $\xi_{1}$ its strongly positive eigenfunction associated.

Lemma 3.2. Let $\Lambda \subset \mathbb{R}$ be a compact interval such that $\lambda<\gamma_{1}$ for all $\lambda \in \Lambda$. Then, there exists $\delta>0$ such that $\Phi_{\lambda}^{t}(u) \neq 0 \forall u \in \mathcal{C}_{\Gamma_{0}}(\bar{\Omega})$ with $\|u\|_{\mathcal{C}(\bar{\Omega})}=\|u\| \in(0, \delta), \forall \lambda \in \Lambda$ and $\forall t \in[0,1]$.

Proof: Suppose the contrary, that there exist $\lambda_{n}, t_{n} \in \mathbb{R}$ and $u_{n} \in \mathcal{C}_{\Gamma_{0}}(\bar{\Omega})$ such that $\lambda_{n} \rightarrow \bar{\lambda}, t_{n} \rightarrow \bar{t},\left\|u_{n}\right\| \rightarrow 0$ and $\Phi_{\lambda_{n}}^{t_{n}}\left(u_{n}\right)=0$. By Lemma 3.1, $u_{n} \geq 0$ and dividing by $\left\|u_{n}\right\|$ we obtain

$$
v_{n}=t_{n} K_{1}\left(\frac{\lambda_{n} M\left(u_{n}\right)+F\left(u_{n}\right)}{\left\|u_{n}\right\|}\right)+t_{n} K_{2}\left(\frac{\lambda_{n} R\left(\gamma\left(u_{n}\right)\right)+G\left(\gamma\left(u_{n}\right)\right)}{\left\|u_{n}\right\|}\right),
$$

where $v_{n}=\frac{u_{n}}{\left\|u_{n}\right\|}$. Thanks to (11) we have that the terms inside $K_{1}$ and $K_{2}$ are uniformly bounded in $\bar{\Omega}$ and on $\Gamma_{1}$, respectively. Since $K_{1}$ and $K_{2}$ are compact operators, then the sequence $v_{n}$ is a relatively compact in $\mathcal{C}(\bar{\Omega})$. Therefore, we can suppose that $v_{n} \rightarrow \bar{v}$ in $\mathcal{C}(\bar{\Omega})$. By (11), we have

$$
\frac{F\left(u_{n}\right)}{\left\|u_{n}\right\|} \rightarrow 0 \text { in } \mathcal{C}(\bar{\Omega}), \quad \frac{G\left(\gamma\left(u_{n}\right)\right)}{\left\|u_{n}\right\|} \rightarrow 0 \text { on } \mathcal{C}\left(\Gamma_{1}\right) .
$$

Passing to the limit in (12), we conclude that

$$
\bar{v}=\bar{t}\left[\bar{\lambda} K_{1}(M(\bar{v}))+\bar{\lambda} K_{2}(R(\gamma(\bar{v})))\right] .
$$

Thanks to $u_{n} \geq 0,\left\|v_{n}\right\|=1$ and by the maximum principle, $\bar{v}$ is a strongly positive function in $\Omega$. Due this fact $\bar{\lambda} \bar{t}=\gamma_{1}$ but this is not possible because $\bar{\lambda} \bar{t}<\gamma_{1}$ by the choice of the set $\Lambda$.

We are going to use the following notation: for $R>0$, let $B_{R}=\{u \in$ $\left.\mathcal{C}_{\Gamma_{0}}(\bar{\Omega}):\|u\|<R\right\}$. Then, $\operatorname{deg}\left(\Phi_{\lambda}, B_{R}, 0\right)$ stands for the degree of $\Phi_{\lambda}$ on $B_{R}$ with respect to 0 , and $i\left(\Phi_{\lambda}, u_{0}, 0\right)$ denotes the index of the solution $u_{0}$ of the equation $\Phi_{\lambda}(u)=0$.

Corollary 3.1. If $\lambda<\gamma_{1}$, then $i\left(\Phi_{\lambda}, 0,0\right)=1$. 
Proof: If $\lambda>0$ consider the interval $\Lambda=[0, \lambda]$ in the contrary case consider $\Lambda=[\lambda, 0]$. Thanks to the Lemma 3.2, we know that $\exists \delta>0$ such that $\forall u \in \mathcal{C}_{\Gamma_{0}}(\bar{\Omega})$ with $\|u\| \in(0, \delta)$ we have $\Phi_{\lambda}^{t}(u) \neq 0, \forall t \in[0,1]$. Therefore by homotopy invariance of the degree we obtain

$$
i\left(\Phi_{\lambda}, 0,0\right)=\operatorname{deg}\left(\Phi_{\lambda}^{1}=\Phi_{\lambda}, B_{\delta}, 0\right)=\operatorname{deg}\left(\Phi_{\lambda}^{0}=I, B_{\delta}, 0\right)=1 .
$$

Lemma 3.3. Let $\lambda>\gamma_{1}$. Then, there exists $\delta>0$ such that $\forall u \in \mathcal{C}_{\Gamma_{0}}(\bar{\Omega})$ with $\|u\| \in(0, \delta), \Phi_{\lambda}(u) \neq \tau \xi_{1}, \forall \tau \geq 0$.

Proof: Assume that there exist sequences $\tau_{n} \geq 0, u_{n} \in \mathcal{C}_{\Gamma_{0}}(\bar{\Omega})$ such that $\left\|u_{n}\right\| \rightarrow 0$ and $\Phi_{\lambda}\left(u_{n}\right)=\tau_{n} \xi_{1}$. Thanks to Proposition 3.1 and similar arguments that we have employed in Lemma 3.1, we have that $u_{n}>0$ is a classical solution of the problem

$$
\begin{cases}L u_{n}=\lambda m(x) u_{n}+f\left(x, u_{n}\right)+\gamma_{1} \tau_{n} m(x) \xi_{1} & \text { in } \Omega \\ u_{n}=0 & \text { on } \Gamma_{0} \\ \mathcal{B} u_{n}=\lambda r(x) u_{n}+g\left(x, u_{n}\right)+\gamma_{1} \tau_{n} r(x) \xi_{1} & \text { on } \Gamma_{1}\end{cases}
$$

Since by Remark 3.1 we can assume that $(b, c)>0$, positive constants are supersolutions of $(L, B, \Omega)$, and so by Proposition 2.1 it follows that $\sigma_{1}[L, B]>0$, and so that by Lemma $2.1, \gamma_{1}>0$. Thanks to conditions (11), we obtain

$$
\begin{cases}L u_{n}>\lambda m(x) u_{n}-\varepsilon u_{n} & \text { in } \Omega, \\ u_{n}=0 & \text { on } \Gamma_{0}, \\ \mathcal{B} u_{n}>\lambda r(x) u_{n}-\varepsilon u_{n} & \text { on } \Gamma_{1},\end{cases}
$$

Hence, $u_{n}$ is strict positive supersolution of $(L-\lambda m(x)+\varepsilon, B-\lambda r(x)+\varepsilon, \Omega)$, and then

$$
\delta_{\varepsilon}(\lambda)=\sigma_{1}[L-\lambda m(x)+\varepsilon, B-\lambda r(x)+\varepsilon]>0 .
$$

On the other hand, we know that $\gamma_{1}$ is the unique zero of the continuous and decreasing function $\delta(\lambda)=\sigma_{1}[L-\lambda m(x), B-\lambda r(x)]$. Since $\lambda>\gamma_{1}$ then $\delta(\lambda)<0$. Moreover, by Proposition 2.1, we infer that exists $\varepsilon>0$ such that $\delta_{\varepsilon}(\lambda)<0$, contradicting $(13)$.

Corollary 3.2. If $\lambda>\gamma_{1}$, then $i\left(\Phi_{\lambda}, 0,0\right)=0$.

Proof: Let $\varepsilon \in(0, \delta)$ where $\delta$ is given Lemma 3.3. Since $\Phi_{\lambda}$ is bounded on $\bar{B}_{\varepsilon}$, then by Lemma 3.3 , there exists $a>0$ such that $\Phi_{\lambda}(u) \neq t a \xi_{1}$, $\forall u \in \bar{B}_{\varepsilon}, \forall t \in[0,1]$. Hence,

$$
i\left(\Phi_{\lambda}, 0,0\right)=\operatorname{deg}\left(\Phi_{\lambda}, B_{\varepsilon}, 0\right)=\operatorname{deg}\left(\Phi_{\lambda}-a \xi_{1}, B_{\varepsilon}, 0\right)=0 .
$$


Let $\mathcal{C} \subset \mathbb{R} \times \mathcal{C}_{\Gamma_{0}}(\bar{\Omega})$ be the closure of the set of positive solutions of (8). Then,

Theorem 3.1. Assume that $(m, r)>0$, (7), (9) and (11). $\gamma_{1}$ is a bifurcation point from the trivial solution, and it is the only one for positive solutions. Moreover, there exists an unbounded continuum $\mathcal{C}_{0} \subset \mathcal{C}$ of positive solutions emanating from $\left(\gamma_{1}, 0\right)$.

Proof: The result follows by Corollaries 3.1 and 3.2 and Ref. ${ }^{5}$, Proposition 3.5. We only remark that the uniqueness of $\gamma_{1}$ follows with the same kind of arguments as in the proof of Lemma 3.2.

\section{Remark 3.3.}

(1) Assume that there exist constants $c_{1}, c_{2} \in \mathbb{R}$ such that

$$
\lim _{s \rightarrow 0^{+}} \frac{f(x, s)}{s}=c_{1} \text { unif. in } \bar{\Omega}, \quad \lim _{s \rightarrow 0^{+}} \frac{g(x, s)}{s}=c_{2} \text { unif. on } \Gamma_{1} .
$$

Then, we can apply the above result to the problem $L_{1} u=\lambda m(x) u+$ $f_{1}(x, u)$ in $\Omega, u=0$ on $\Gamma_{0}$ and $\mathcal{B}_{2} u=\lambda r(x) u+g_{2}(x, u)$ on $\Gamma_{1}$, where $L_{1}=L-c_{1}, \mathcal{B}_{2}=\mathcal{B}-c_{2}, f_{1}(x, u)=f(x, u)-c_{1} u$ and $g_{2}(x, u)=g(x, u)-c_{2} u$, and so $f_{1}$ and $g_{2}$ satisfy $(11)$

(2) The case that $m>0, r \equiv 0$ (i.e. the bifurcation parameter only in the equation) can be included in the Theorem 3.1. Indeed, if $b \geq 0$ then ( 7$)$ is verified. If $b<0$ or changes sign we can perform a change $u=e^{M \psi} v$ where $\psi$ is the function that appears on Ref. ${ }^{20}$, Proposition 3.4, and the original problem is transformed into a similar new problem where the new $b$, say $\widetilde{b}>0$.

(3) It is also possible to cover the case $m \equiv 0, r>0$ (i.e. the bifurcation parameter only at the boundary). Indeed, if $\sigma_{1}[L, D] \leq 0$ then it can be proved that bifurcation from the trivial solution does not occur. Now, assume $\sigma_{1}[L, D]>0$. By Proposition 2.1 there exists $\mu r$ with $\mu$ enough big such that $\sigma_{1}[L, B+\mu r]>0$. Then, there exists a unique solution $h>0$ in $\bar{\Omega}$ of the problem

$$
\begin{cases}L h=1 & \text { in } \Omega, \\ h=1 & \text { on } \Gamma_{0}, \\ (\mathcal{B}+\mu r) h=0 & \text { on } \Gamma_{1} .\end{cases}
$$

Now, we perform the change $u=h v$, which transforms the original problem into a new problem where the new $c, \widetilde{c}>0$. 
(4) A similar result can be obtained for bifurcation from infinity.

(5) We have not found in the literature a general result similar to Theorem 3.1. In Ref. ${ }^{27}$ the author studied bifurcation form infinity for a similar equation with nonlinearities asymptotically linear. In Ref. ${ }^{7}$ the bifurcation method is studied but with nonlinearities only at the boundary. In both papers, $L u=-\Delta u+u$ and $b(x) \geq 0$.

In the rest of the section we consider the problem

$$
\begin{cases}L u=\lambda f(x, u) & \text { in } \Omega, \\ u=0 & \text { on } \Gamma_{0}, \\ \mathcal{B} u=g(x, u) & \text { on } \Gamma_{1},\end{cases}
$$

where $f \in \mathcal{C}^{\alpha}(\bar{\Omega} \times \mathbb{R}), g \in \mathcal{C}^{1, \alpha}\left(\Gamma_{1} \times \mathbb{R}\right)$. Throughout the rest of the section we assume the following conditions $(c, b)>0,(9)$ and

$$
\begin{gathered}
\lim _{s \rightarrow 0^{+}} \frac{f(x, s)}{s}=+\infty \text { uniformly in } \bar{\Omega}, \\
\lim _{s \rightarrow 0^{+}} \frac{g(x, s)}{s}=0 \text { uniformly on } \Gamma_{1} .
\end{gathered}
$$

We have that $u$ is a classical nonnegative solution of (14) if and only if $\Psi_{\lambda}(u)=0$ in $\mathcal{C}_{\Gamma_{0}}(\bar{\Omega})$, where $\Psi_{\lambda}: \mathcal{C}_{\Gamma_{0}}(\bar{\Omega}) \rightarrow \mathcal{C}_{\Gamma_{0}}(\bar{\Omega})$ is defined as

$$
\Psi_{\lambda}(u)=u-K_{1}\left(\lambda F\left(u^{+}\right)\right)-K_{2}\left(G\left(\gamma\left(u^{+}\right)\right)\right) .
$$

Consider $\Psi_{\lambda}^{t}(u)=u-t K_{1}\left(\lambda F\left(u^{+}\right)\right)-t K_{2}\left(G\left(\gamma\left(u^{+}\right)\right)\right)$.

Lemma 3.4. If $\lambda<0$ then there exists $\delta>0$ such that $\forall u \in \mathcal{C}_{\Gamma_{0}}(\bar{\Omega})$ with $\|u\|_{\mathcal{C}(\bar{\Omega})}=\|u\| \in(0, \delta)$ we have $\Psi_{\lambda}^{t}(u) \neq 0, \forall t \in[0,1]$.

Proof: Suppose the contrary, then there exist sequences $t_{n} \in \mathbb{R}, u_{n} \in$ $\mathcal{C}_{\Gamma_{0}}(\bar{\Omega})$ such that $t_{n} \rightarrow \bar{t},\left\|u_{n}\right\| \rightarrow 0$ with $\Psi_{\lambda}^{t_{n}}\left(u_{n}\right)=0$. Dividing by $\left\|u_{n}\right\|$, we obtain

$$
v_{n}=\lambda t_{n} K_{1}\left(\frac{F\left(u_{n}\right)}{\left\|u_{n}\right\|}\right)+t_{n} K_{2}\left(\frac{G\left(\gamma\left(u_{n}\right)\right)}{\left\|u_{n}\right\|}\right),
$$

where $v_{n}=\frac{u_{n}}{\left\|u_{n}\right\|}$. Since $\lambda<0$, the fact that $\left\|u_{n}\right\| \geq\left\|u_{n}\right\|_{\Gamma_{1}}$ and using (15) and (16) we get that $v_{n} \rightarrow 0$ in $\mathcal{C}(\bar{\Omega})$, a contradiction because $\left\|v_{n}\right\|=1$.

Lemma 3.5. If $\lambda>0$ then there exists $\delta>0$ such that $\forall u \in \mathcal{C}_{\Gamma_{0}}(\bar{\Omega})$ with $\|u\| \in(0, \delta)$ and $\forall \tau \geq 0$ we have $\Psi_{\lambda}(u) \neq \tau \varphi_{1}$, where $\varphi_{1}$ is a positive eigenfunction associated to $\sigma_{1}[L, B]$. 
Proof: Let us assume that for some sequence $u_{n} \in \mathcal{C}_{\Gamma_{0}}(\bar{\Omega})$ with $\left\|u_{n}\right\| \rightarrow 0$ and numbers $\tau_{n} \geq 0, \Psi_{\lambda}\left(u_{n}\right)=\tau_{n} \varphi_{1}$. It is clear, by the maximum principle, that $u_{n}>0$ and it is a classical solution of the problem

$$
\begin{cases}L u_{n}=\lambda f\left(x, u_{n}\right)+\sigma_{1}[L, B] \tau_{n} \varphi_{1} & \text { in } \Omega, \\ u_{n}=0 & \text { on } \Gamma_{0}, \\ \mathcal{B} u_{n}=g\left(x, u_{n}\right) & \text { on } \Gamma_{1} .\end{cases}
$$

Take $\varepsilon>0$, and $M>\sigma_{1}[L, B+\varepsilon]$. Since $\sigma_{1}[L, B]>0$ and due to $u_{n} \rightarrow 0$ in $\mathcal{C}(\bar{\Omega})$ we have, using (15) and (16), that there exists $n_{0}$ such that $\forall n \geq n_{0}$

$$
\begin{cases}L u_{n}=\lambda f\left(x, u_{n}\right)+\sigma_{1}[L, B] \tau_{n} \varphi_{1}>M u_{n} & \text { in } \Omega, \\ u_{n}=0 & \text { on } \Gamma_{0}, \\ \mathcal{B} u_{n}=g\left(x, u_{n}\right)>-\varepsilon u_{n} & \text { on } \Gamma_{1} .\end{cases}
$$

Therefore, $u_{n}$ is a positive strict supersolution of $(L-M, B+\varepsilon, \Omega)$, then $\sigma_{1}[L-M, B+\varepsilon]>0$, and so $M<\sigma_{1}[L, B+\varepsilon]$, a contradiction.

Theorem 3.2. Under conditions $(c, b)>0,(9),(15)$ and (16), $\lambda=0$ is a bifurcation point from trivial solution and it is the only one for positive solutions. Moreover, there exists an unbounded continuum $\mathcal{C}_{0}$ of positive solutions emanating from $(0,0)$.

Proof: It is possible, thanks to Lemmas 3.4 and 3.5, reasoning as Theorem 3.1 to prove that there exists an unbounded continuum $\mathcal{C}_{0}$. We only need to prove uniqueness of bifurcation point. By Lemma 3.4 we can prove that bifurcation from the trivial solution does not occur for points of the form $\left(\lambda_{0}, 0\right), \lambda_{0}<0$. Let us assume that there exists a sequence $\left(\lambda_{n}, u_{\lambda_{n}}\right) \in \mathbb{R} \times \mathcal{C}_{\Gamma_{0}}(\bar{\Omega})$ verifying $\left(\lambda_{n}, u_{\lambda_{n}}\right) \rightarrow\left(\lambda_{0}, 0\right)$ in $\mathbb{R} \times \mathcal{C}_{\Gamma_{0}}(\bar{\Omega})$ with $\lambda_{0}>0$. Then

$$
L u_{\lambda_{n}}=\lambda_{n} f\left(x, u_{\lambda_{n}}\right)>\lambda_{n} M u_{\lambda_{n}}, \quad \mathcal{B} u_{\lambda_{n}}=g\left(x, u_{\lambda_{n}}\right)>-\varepsilon u_{\lambda_{n}} .
$$

At this point we only need to follow the reasoning of Lemma 3.5 to obtain a contradiction.

\section{Remark 3.4.}

(1) A similar result is obtained under the condition

$$
f(x, s)=m(x) f(s),
$$

with $m \in \mathcal{C}^{\alpha}(\bar{\Omega}), m(x) \geq 0$, and non-trivial, $f \in \mathcal{C}^{\alpha}(\mathbb{R})$ and $\lim _{s \rightarrow 0^{+}} \frac{f(s)}{s}=+\infty$, instead of (15). 
(2) Simliar results still are true for equations of the form

$$
\begin{cases}L u=h(x, u) & \text { in } \Omega, \\ u=0 & \text { on } \Gamma_{0}, \\ \mathcal{B} u=\lambda i(x, u) & \text { on } \Gamma_{1},\end{cases}
$$

where $h$ and $i$ play the same role as $g$ and $f$, respectively.

\section{Stability, uniqueness and a-priori bounds}

In this section we present (without proofs) some results concerning to the stability, uniqueness and a-priori bounds of the solutions of the problem

$$
\begin{cases}L u=f(x, u) & \text { in } \Omega, \\ u=0 & \text { on } \Gamma_{0}, \\ \mathcal{B} u=g(x, u) & \text { on } \Gamma_{1},\end{cases}
$$

where $f$ and $g$ are regular functions.

Let $u$ a non-negative solution of (18). For the study of the stability of $u$, we linearize (18) around $u$ and consider the eigenvalue problem:

$$
\begin{cases}L w=f_{u}(x, u) w+\gamma(u) w & \text { in } \Omega, \\ w=0 & \text { on } \Gamma_{0}, \\ \mathcal{B} w=g_{u}(x, u) w+\gamma(u) w & \text { on } \Gamma_{1} .\end{cases}
$$

Thanks to Theorem 2.1, we know that the eigenvalue problem has a unique principal eigenvalue $\gamma_{1}(u)=\gamma_{1}\left[L-f_{u}(x, u), B-g_{u}(x, u)\right]$.

Theorem 4.1. Let u a nonnegative solution of (18).

(1) If $\gamma_{1}(u)>0$, then $u$ is linearly asymptotically stable (l. a. s.).

(2) If $\gamma_{1}(u)<0$, then $u$ is unstable.

In general, determinate the sign of $\gamma_{1}(u)$ is not easy. Due this fact, we give the following characterization using the following related problem:

$$
\begin{cases}L w=f_{u}(x, u) w & \text { in } \Omega, \\ w=0 & \text { on } \Gamma_{0}, \\ \mathcal{B} w=g_{u}(x, u) w & \text { on } \Gamma_{1} .\end{cases}
$$

Using Lemma 2.1 and Proposition 2.1, we get

Theorem 4.2. $\gamma_{1}(u)>0$ (resp. $\left.\gamma_{1}(u)<0\right)$ if and only if the problem (20) admits a positive strict supersolution (resp. subsolution). 
With respect to the uniqueness, we have:

Theorem 4.3. Assume $f \in \mathcal{C}^{1}(\bar{\Omega} \times[0,+\infty))$ and $g \in \mathcal{C}^{1}\left(\Gamma_{1} \times[0,+\infty)\right)$ and that

$$
t \mapsto \frac{f(x, t)}{t}, \quad t \mapsto \frac{g(x, t)}{t} \quad \text { are nonincreasing functions in } t>0,
$$

and at least one of them is a decreasing function. Then, problem (18) admits at most one positive solution.

We assume $f \in \mathcal{C}(\bar{\Omega} \times[0,+\infty)), g \in \mathcal{C}^{1, \alpha}\left(\Gamma_{1} \times[0,+\infty)\right)$ and there exists $p \in\left(1, \frac{N+2}{N-2}\right), q \in\left(1, \frac{N}{N-2}\right)$ that verifies

$$
\lim _{t \rightarrow+\infty} \frac{f(x, t)}{t^{p}}=h(x),
$$

uniformly in $\bar{\Omega}$ with $h \in \mathcal{C}(\bar{\Omega})$ a positive function and

$$
\lim _{t \rightarrow+\infty} \frac{g(x, t)}{t^{q}}=i(x)
$$

uniformly on $\Gamma_{1}$ with $i \in \mathcal{C}^{1, \alpha}(\bar{\Omega})$ a positive function.

Theorem 4.4. Let $u \in \mathcal{C}^{2}(\Omega) \cap \mathcal{C}^{1}(\bar{\Omega})$ a nonnegative solution of the problem (18). Suppose that one of the following conditions is satisfied:

(1) (21), (22) and $p \neq 2 q-1$;

(2) The maximum of $u$ is attained on $\partial \Omega$, (22), (21) is satisfied for any function $h$ and $p<2 q-1$.

Then, there exists $C(p, q, \Omega)$ is a positive constant depending on $p, q$ and $\Omega$ such that for all $x \in \bar{\Omega}$

$$
u(x) \leq C(p, q, \Omega)
$$

\section{Remark 4.1.}

(1) The condition $p \neq 2 q-1$ appears in other papers, see Ref. ${ }^{12}$ and it is necessary to apply a Gidas-Spruck argument.

(2) The proofs of Theorems 4.1, 4.3 and 4.4 can be found in Ref. ${ }^{23}$. Theorem 4.1 complements and improves Theorem 5.6.2 of Ref. ${ }^{25}$ and Theorem 3.1 of Ref. ${ }^{28}$. Theorem 4.3 is proved in Ref. ${ }^{24}$ where other uniqueness' results can be found. Finally, a-priori results have been shown in Ref. ${ }^{30}$ with nonlinearities only at the boundary (see also Ref. ${ }^{12}$ for systems) and Ref. ${ }^{14}$ for particular nonlinearities in the equation and on the boundary. 


\section{Some applications}

In this section we are going to study some equations with nonlinear boundary. The first equation is

$$
\begin{cases}L u=\lambda u & \text { in } \Omega, \\ u=0 & \text { on } \Gamma_{0}, \\ \mathcal{B} u=a(x) u^{r} & \text { on } \Gamma_{1} .\end{cases}
$$

where $r>1$ and $a \in \mathcal{C}^{1, \alpha}\left(\Gamma_{1}\right)$.

\section{Theorem 5.1.}

(1) Assume that $a<0$. (23) has a positive solution if and only if $\sigma_{1}[L, B]<\lambda<\sigma_{1}[L, D]$. Moreover, if the solution exists, it is unique and l. a. s.

(2) Assume that $a>0$. If $u$ is a positive solution of (23) then, $\lambda<$ $\sigma_{1}[L, B]$. If $1<r<\frac{N}{N-2}$ then there exists at least a positive solution of (23) for all $\lambda<\sigma_{1}[L, B]$. Moreover, all positive solutions of (23) are unstable.

Proof: (1) Assume $a<0$. Suppose $u$ is a nonnegative solution of (23), then, by the strong maximum principle, $u$ is strongly positive, and so, $\lambda=\sigma_{1}\left[L, B-a(x) u^{r-1}\right]$. Applying Proposition 2.1, we obtain

$$
\sigma_{1}[L, B]<\lambda=\sigma_{1}\left[L, B-a(x) u^{r-1}\right]<\sigma_{1}[L, D] .
$$

Now, we construct a sub-supersolution for the problem (23). Fix $\lambda_{0} \in$ $\left(\sigma_{1}[L, B], \sigma_{1}[L, D]\right)$. By Proposition 2.1 there exist $k_{1}$ and $k_{2}$ such that $\sigma_{1}\left[L, B+k_{2}\right]>\lambda_{0}>\sigma_{1}\left[L, B+k_{1}\right]$. Now, the pair $\underline{u}=\varepsilon \varphi_{1}$ and $\bar{u}=M \varphi_{2}$ with $\varepsilon$ little and $M$ large enough, and $\varphi_{i}$ a strongly positive eigenfunction associated to $\sigma_{1}\left[L, B+k_{i}\right]$, is a sub-supersolution of (23).

Uniqueness follows by Theorem 4.3. For the stability we use Theorem 4.2. Choose $\bar{u}=u$ with $u$ solution of (23) then, $(L-\lambda) \bar{u}=0$ in $\Omega$, and

$$
\bar{u}=0 \quad \text { on } \Gamma_{0}, \quad\left(\mathcal{B}-a(x) r u^{r-1}\right) \bar{u}>\mathcal{B} u-a(x) u^{r}=0 \quad \text { on } \Gamma_{1},
$$

i. e. $u$ is a positive strict supersolution of the linearized problem around $u$, $\left(L-\lambda, B-r a(x) u^{r-1}, \Omega\right)$.

(2) Assume now that $a>0$. If $u$ is a nonnegative solution of (23) then $\lambda=\sigma_{1}\left[L, B-a(x) u^{r-1}\right]<\sigma_{1}[L, B]$. By Theorem 3.1, there exists a unbounded continuum $\mathcal{C}_{0}$ emanating from $\left(\sigma_{1}[L, B], 0\right)$. Its direction is subcritical by the limitation of $\lambda$ and, under condition $1<r<\frac{N}{N-2}$, 
Theorem 4.4 proves us that the projection of $\mathcal{C}_{0}$ on $\lambda$-axis $\mathcal{P}_{\lambda}\left(\mathcal{C}_{0}\right)=$ $\left(-\infty, \sigma_{1}[L, B]\right)$. Positive solutions of $(23)$ are unstable because if $u$ is a positive solution then,

$$
\sigma_{1}\left[L-\lambda, B-r a(x) u^{r-1}\right]<\sigma_{1}\left[L-\lambda, B-a(x) u^{r-1}\right]=0 .
$$

Remark 5.1. In the case $a<0$ and thanks to the subsolution that we have built, it could be proved that for $\mathcal{K}$ a compact subset of $\bar{\Omega} \backslash \Gamma_{0}$,

$$
\lim _{\lambda \rightarrow \sigma_{1}[L, D]^{-}} \min _{\mathcal{K}} u_{\lambda}=+\infty .
$$

\subsection{Elliptic equation with a logistic term at the boundary}

From the results obtained of the equation (23), we can deduce results for the equation (3).

\section{Theorem 5.2.}

(1) If $\sigma_{1}[L, D] \leq 0$, (3) does not have positive solutions.

(2) Assume $\sigma_{1}[L, D]>0$.

(a) If $a<0$, then (3) has positive solutions if and only if

$$
\mu>\lambda_{1}[L, B] .
$$

Moreover, if the positive solution exists, is unique and $l . a . s$.

(b) If $a>0$ and there exists a positive solution of (3), then $\mu<\lambda_{1}[L, B]$. Moreover, under condition $1<r<\frac{N}{N-2}$, there exists at least one positive solution if $\mu<\lambda_{1}[L, B]$. Furthermore, positive solutions of (3) are unstable.

Proof: We only need to put $\lambda=0$ and $\widetilde{b}(x)=b(x)-\mu$ in Theorem 5.1. If $a<0,(3)$ possesses a positive solution if and only if $\sigma_{1}[L, B-\mu]<0<$ $\sigma_{1}[L, D]$. By the definition of $\lambda_{1}[L, B]$, the result follows. Analogously the case $a>0$.

\subsection{A sublinear-superlinear equation}

Now, we study the equation (4).

Theorem 5.3. $(0,0)$ is the unique point of bifurcation from the trivial solution, and there exists an unbounded continuum $\mathcal{C}_{0}$ of positive solutions emanating from $(0,0)$. Moreover, 
(1) Respect bifurcation direction:

(a) If $p<r$ (resp. $p>r$ ) then bifurcation direction is supercritical (resp. subcritical).

(b) If $p=r$ bifurcation direction is supercritical (resp. subcritical) for $|\Omega|>|\partial \Omega|$ (resp. $|\Omega|<|\partial \Omega|)$.

(2) If $p=r$ and $|\Omega| \leq|\partial \Omega|$, (4) does not have positive solutions for $\lambda \geq 0$

(3) If $p<2 r-1$, (4) does not have positive solutions for $\lambda$ large enough.

(4) If $p \leq r$ and $\lambda \leq 0$ every positive solution is unstable.

(5) If $p<2 r-1$ and $r<\frac{N}{N-2}$ then every positive solution is bounded in $L^{\infty}$ norm.

(6) If $p>2 r-1$, there exists solution for all $\lambda \geq 0$.

Proof: Due to Theorem 3.1, we have a unbounded continuum $\mathcal{C}_{0}$ of positive (4) emanating from $\left(\sigma_{1}[-\Delta, \mathcal{N}]=0,0\right)$. We study the bifurcation direction. Consider $\lambda_{n} \rightarrow \sigma_{1}[-\Delta, \mathcal{N}]$ and its solutions associated $u_{n}$. Then, multiplying the equation by $\varphi_{1}=c>0$, the eigenfunction associated to the eigenvalue $\sigma_{1}[-\Delta, \mathcal{N}]$, we obtain

$$
\left(\sigma_{1}[-\Delta, \mathcal{N}]-\lambda_{n}\right) \int_{\Omega} u_{n} \varphi_{1} d x=\int_{\partial \Omega} u_{n}^{r} \varphi_{1} d \sigma-\int_{\Omega} u_{n}^{p} \varphi_{1} d x .
$$

Assume for example that $p<r$, multiply $(24)$ by $\left\|u_{n}\right\|_{\mathcal{C}(\bar{\Omega})}^{-p}$ and taking into account that $\frac{u_{n}}{\left\|u_{n}\right\|_{\mathcal{C}(\bar{\Omega})}} \rightarrow \varphi_{1}$ in $\mathcal{C}(\bar{\Omega})$ (see the proof of Lemma 3.2), it follows

$$
S g\left(\sigma_{1}[-\Delta, \mathcal{N}]-\lambda_{n}\right)=S g\left(-\int_{\Omega} \varphi_{1}^{p+1} d x\right),
$$

hence, $\sigma_{1}[-\Delta, \mathcal{N}]<\lambda_{n}$. All results related to local bifurcation can be proved by the same way.

Let $u$ a positive solution of (4) with $p=r$. Then, if we multiply the equation (4) by $1 / u^{r}$, and integrating by parts, we get

$$
-r \int_{\Omega} u^{-r-1}|\nabla u|^{2}-|\partial \Omega|+|\Omega|=\lambda \int_{\Omega} u^{1-r}
$$

Then, paragraph (2) follows.

Assume that the problem (4) has a positive solution for every $\lambda>0$. Consider the parabolic problem

$$
\begin{cases}w_{t}-\Delta w=-w^{p} & \text { in } \Omega \times(0, T), \\ \frac{\partial w}{\partial n}=w^{r} & \text { on } \partial \Omega \times(0, T), \\ w(x, 0)=w_{0} & \text { in } \Omega .\end{cases}
$$


We know by Ref. ${ }^{6}$, Theorem 2.3 , that if $p<2 r-1$ then all positive solutions of (25) blow-up in finite time for $w_{0}$ with large $L^{\infty}$ norm. Take $u_{\lambda}$ a solution of (4), if we prove that $u_{\lambda}$ is supersolution of (25) for large $\lambda$, then $u_{\lambda}(x)>w(x, t)$ for all $t \in(0, T)$ which is a contradiction. In order to prove this, we only need that $u_{\lambda}>w_{0}$. It is clear that for $\lambda>0 u_{\lambda}$ is supersolution of the problem

$$
\begin{cases}-\Delta v=\lambda v-v^{p} & \text { in } \Omega \\ \frac{\partial v}{\partial n}=0 & \text { on } \partial \Omega .\end{cases}
$$

As solutions of (26) are, for $\lambda>0, \lambda^{1 /(p-1)}$ then

$$
u_{\lambda}>\lambda^{1 /(p-1)} \text {. }
$$

Now, there exists $\lambda>0$ large enough such that $\left\|w_{0}\right\|_{\infty}<\lambda^{1 /(p-1)}<u_{\lambda}$, this concludes paragraph (3).

Let $u$ a positive solution, we are going to prove that under condition $p \leq r$ this solution is unstable. For that, thanks to Theorem 4.2, we have to show that

$$
\sigma_{1}\left[-\Delta-\lambda+p u^{p-1}, \mathcal{N}-r u^{r-1}\right]<0 .
$$

For this fact we choose as subsolution, $\underline{u}=u^{q}$, where $q$ will be fixed later. We have that

$$
\frac{\partial \underline{u}}{\partial n}-r u^{r-1} \underline{u}=(q-r) u^{q+r-1} \quad \text { on } \partial \Omega
$$

and in $\Omega$,

$$
\left(-\Delta-\lambda+p u^{p-1}\right) \underline{u}=q(1-q) u^{q-2}|\nabla u|^{2}+\lambda u^{q}(q-1)+u^{p+q-1}(p-q) .
$$

Choosing $q$ such that $p \leq q \leq r$, it follows (28), so that paragraph (4).

By (27), $u$ attains its maximum on $\partial \Omega$. So, paragraph (5) follows by Theorem 4.4 .

For the last paragraph we only need to find a sub-supersolution of (4) for every $\lambda \geq 0$. We choose as subsolution ${ }^{\mathrm{a}}$

$$
\underline{u}=\varepsilon e^{-\delta \phi_{1}}
$$

where $\varepsilon, \delta>0$ can be chosen later and $\phi_{1}$ is the positive eigenfunction associated to $\sigma_{1}=\sigma_{1}[-\Delta, D]$ with $\left\|\phi_{1}\right\|_{\infty}=1 / 2$. After some calculations we obtain

$$
\nabla \underline{u}=-\varepsilon \delta e^{-\delta \phi_{1}} \nabla \phi_{1}, \quad \Delta \underline{u}=-\varepsilon \delta e^{-\delta \phi_{1}}\left(-\delta\left|\nabla \phi_{1}\right|^{2}+\Delta \phi_{1}\right) .
$$

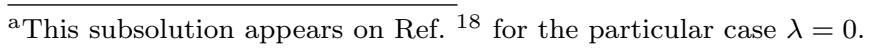


Thanks to Hopf's Lemma, it follows that

$$
\max _{x \in \partial \Omega}\left|\frac{\partial \phi_{1}}{\partial n}\right|=C_{1}, \quad C_{1}>0 .
$$

Since, $\phi_{1}=0$ on $\partial \Omega$, we only need to verify on the boundary that

$$
\delta C_{1} \leq \varepsilon^{r-1} .
$$

In the equation we must check that

$$
-\delta^{2}\left|\nabla \phi_{1}\right|^{2}-\delta \sigma_{1} \phi_{1}+\varepsilon^{p-1} e^{-\delta \phi_{1}(p-1)} \leq \lambda .
$$

Observe that if $\lambda>0$, we only need to choose $\varepsilon$ and $\delta$ positive and small enough for that (30) and (31) hold. So that, we are going to study the case $\lambda=0$. From (30) we choose $\delta=\frac{\varepsilon^{r-1}}{C_{1}}$, so that (31) transforms into

$$
-\frac{\varepsilon^{2(r-1)}}{C_{1}^{2}}\left|\nabla \phi_{1}\right|^{2}-\frac{\varepsilon^{r-1}}{C_{1}} \sigma_{1} \phi_{1}+\varepsilon^{p-1} e^{-\frac{\varepsilon^{r-1}}{C_{1}} \phi_{1}(p-1)} \leq 0 .
$$

Now, due to $\phi_{1}=0$ on $\partial \Omega$, but on the boundary $\partial \phi_{1} / \partial n<0$, there exist some constants $C_{2}, C_{3}>0$ such that

$$
\left|\nabla \phi_{1}\right| \geq C_{2} \quad \text { en } \Omega_{1}:=\left\{x \in \Omega: \phi_{1}(x) \leq C_{3}\right\} .
$$

In this way, in $\Omega_{1}$ for that the condition (32) must be fulfilled we need that $p-1>2(r-1)$ thus $p+1>2 r$.

On the other hand, in $\Omega \backslash \bar{\Omega}_{1}$ we need that $p-1>r-1$.

The supersolution follows by Ref. ${ }^{22}$, it was used also in Ref. ${ }^{18}$, in both cases for the particular case $\lambda=0$. We choose

$$
\bar{u}:=M^{A}\left[2-\left(1-\phi_{1}\right)^{B}\right]^{C},
$$

where $M>0$ will be chosen large and $A, B$ and $C$ will be fixed later. Observe that

$$
\begin{aligned}
\nabla \bar{u}= & B C M^{A}\left[2-\left(1-\phi_{1}\right)^{B}\right]^{C-1}\left(1-\phi_{1}\right)^{B-1} \nabla \phi_{1}, \\
\Delta \bar{u}= & B C M^{A}\left[2-\left(1-\phi_{1}\right)^{B}\right]^{C-2}\left(1-\phi_{1}\right)^{B-2} . \\
& \left\{(C-1)\left(1-\phi_{1}\right)^{B}\left|\nabla \phi_{1}\right|^{2}+\left[2-\left(1-\phi_{1}\right)^{B}\right] \Delta \phi_{1}\left(1-\phi_{1}\right)-\right. \\
& {\left.\left[2-\left(1-\phi_{1}\right)^{B}\right](B-1)\left|\nabla \phi_{1}\right|^{2}\right\} . }
\end{aligned}
$$

Taking into account (29), on the boundary it must be verified (observe that $\phi_{1}=0$ and so that $\left.\left[2-\left(1-\phi_{1}\right)^{B}\right]=1\right)$ :

$$
-B C M^{A(1-r)} \geq \frac{1}{C_{1}} \text {. }
$$


For the equation, we need that

$$
\begin{aligned}
\lambda \leq & -B C(C-1)\left[2-\left(1-\phi_{1}\right)^{B}\right]^{-2}\left(1-\phi_{1}\right)^{2(B-1)}\left|\nabla \phi_{1}\right|^{2} \\
& -B C\left[2-\left(1-\phi_{1}\right)^{B}\right]^{-1} \Delta \phi_{1}\left(1-\phi_{1}\right)^{B-1} \\
& +B C(B-1)\left[2-\left(1-\phi_{1}\right)^{B}\right]^{-1}\left(1-\phi_{1}\right)^{B-2}\left|\nabla \phi_{1}\right|^{2} \\
& +M^{A(p-1)}\left[2-\left(1-\phi_{1}\right)^{B}\right]^{C(p-1)} .
\end{aligned}
$$

Take $A>0, C=-1 / C_{1}$ and $B=M^{b}$, with $b$ to be fixed later. With this choice, for condition (34) it will be needed

$$
b+A(1-r) \geq 0 . \quad(\Longrightarrow b>0) .
$$

Now, we study the term (35). First term in the right hand tends to $-\infty$ or zero (the term $\left(1-\phi_{1}\right)^{2(B-1)}$ can tend to zero). Second term is similar, we remind that $-\Delta \phi_{1}=\sigma_{1}[-\Delta, D] \phi_{1}$. Third term tends to $-\infty$ with order $M^{2 b}$ and the last one to $+\infty$ with order $M^{A(p-1)}$, so we have to impose $A(p-1)>2 b$. This last inequality and (36) are possible because $p+1>2 r$.

Remark 5.2. Except paragraphs (2) and (3), Theorem 5.3 is true for more general operators $L$ and $B$.

\subsection{The concave-convex equation}

Finally, we consider (5). Assume the following conditions

$$
c>c_{0}>0 \text { en } \bar{\Omega}, \text { with } c_{0} \in \mathbb{R} .
$$

We distinguish two different cases: $a$ negative and positive.

Theorem 5.4. Assume that $a<0$. The problem (5) has a positive solution $v_{\lambda}$ if and only if $\lambda>0$. For $\lambda>0$, it is the unique positive solution, it is l. a. s. and

$$
\lim _{\lambda \rightarrow 0^{+}}\left\|v_{\lambda}\right\|_{\infty}=0
$$

Proof: Thanks to the maximum principle (5) does not posses nonnegative solutions for $\lambda \leq 0$. By Theorem 3.2, there exists a continuum $\mathcal{C}_{0}$ emanating from $(0,0)$ supercritically. On the other hand, $\bar{v}=M \varphi_{1}$ is, for $M$ large enough, supersolution of (5) where $\varphi_{1}$ is the positive eigenfunction associated to $\sigma_{1}[L, \mathcal{N}]$. This is true because $\sigma_{1}[L, \mathcal{N}]>0$, which it follows by (37). Since $M$ can be chosen large enough that $M \varphi_{1}>v_{\lambda}$ for $\lambda>0$ small, where $v_{\lambda}$ denotes the solution of the problem founded by bifurcation. Then, we have a family of supersolutions such that a solution belonging to 
the continuum is smaller than the supersolution. Now, adapting the proof of main theorem of Ref. ${ }^{13}$, we have that there exists at least a positive solution for all $\lambda>0$. Finally, for $\lambda>0$

$0=\sigma_{1}\left[L-\lambda m(x) v_{\lambda}^{q-1}, \mathcal{N}-a(x) v_{\lambda}^{r-1}\right]<\sigma_{1}\left[L-\lambda q m(x) v_{\lambda}^{q-1}, \mathcal{N}-r a(x) v_{\lambda}^{r-1}\right]$

so the stability follows. Uniqueness follows by Theorem 4.3.

Theorem 5.5. Assume that $a>0$.

(1) From (0,0) emanates supercritically an unbounded continuum $\mathcal{C}_{0}$ of positive solutions. Moreover, it is the unique bifurcation point from the trivial solution.

(2) There exists $\lambda^{*}>0$ such that for $\lambda>\lambda^{*}$ problem (5) does not have positive solutions.

(3) There exists $\delta>0$ such that there exists at most a positive solution $u_{\lambda}$ of (5) such that $\left\|u_{\lambda}\right\|_{\infty} \leq \delta$.

(4) Moreover, if $L$ is self-adjoint and $r<\frac{N}{N-2}$ then:

(a) $\mathcal{P}_{\lambda}\left(\mathcal{C}_{0}\right)=(-\infty, \Lambda]$, for some $\Lambda<+\infty$.

(b) There exists at least two positive solutions in $(0, \Lambda)$.

(c) There exists a unique positive solution in $(0, \Lambda) l$. a. $s$.

Proof: Since the proof follows the same lines that Theorem 6.9 in Ref. ${ }^{11}$, we only sketch it.

The existence of the continuum $\mathcal{C}_{0}$ follows by Theorem 3.2. We prove now that the bifurcation direction is supercritical. Assume that there exist $\lambda_{n} \leq 0$ and $u_{\lambda_{n}} \in \mathcal{C}(\bar{\Omega}), u_{\lambda_{n}} \geq 0$ such that $\left(\lambda_{n}, u_{\lambda_{n}}\right) \rightarrow(0,0)$ in $\mathbb{R} \times \mathcal{C}(\bar{\Omega})$. Then, for $n \geq n_{0}$ we get

$$
L u_{\lambda_{n}} \leq 0 \quad \text { in } \Omega, \quad \frac{\partial u_{\lambda_{n}}}{\partial n}=a(x) u_{\lambda_{n}}^{r}<\varepsilon a_{M} u_{\lambda_{n}} \quad \text { on } \partial \Omega,
$$

where $a_{M}=\max _{\partial \Omega} a$. On the other hand, since $\sigma_{1}[L, \mathcal{N}]>0$ then, for $\varepsilon>0$ small, $\sigma_{1}\left[L, \mathcal{N}-\varepsilon a_{M}\right]>0$, and applying the strong maximum principle we obtain that $u_{\lambda_{n}} \equiv 0$, a contradiction.

Now, we are going to prove paragraph (2). Suppose that there exists positive solution $u_{\lambda}$ of (5) for all $\lambda$, in particular for $\lambda>1$. Let $v_{1}$ be the unique positive solution of

$$
\begin{cases}L u=m(x) u^{q} & \text { in } \Omega \\ \frac{\partial u}{\partial n}=0 & \text { on } \partial \Omega\end{cases}
$$


Since $u_{\lambda} / \lambda$ is supersolution of (39) for $\lambda>1$, then $u_{\lambda}>\lambda v_{1}$ for $\lambda>1$. On the other hand, since $u_{\lambda}$ is a positive solution of (5), we get

$$
0=\sigma_{1}\left[L-\lambda m(x) u_{\lambda}^{q-1}, \mathcal{N}-a(x) u_{\lambda}^{r-1}\right]<\sigma_{1}\left[L, \mathcal{N}-\lambda^{r-1} a_{0}^{r-1} v_{1}^{r-1}\right],
$$

where $a_{0}=\min _{\partial \Omega} a(x)$. This is an absurdum. Indeed, since $r>1$, we have

$$
\lim _{\lambda \rightarrow+\infty} \sigma_{1}\left[L, \mathcal{N}-\lambda^{r-1} a_{0}^{r-1} v_{1}^{r-1}\right]=-\infty .
$$

Now, define

$$
\Lambda:=\sup \{\lambda \in \mathbb{R}: \text { (5) has a positive solution }\} .
$$

We have proved that $0<\Lambda<+\infty$. Moreover, it is not difficult to prove the existence of a minimal solution $u_{\lambda}$ for all $\lambda \in(0, \Lambda)$.

The following result shows properties of the principal eigenvalue, denoted by $\gamma_{1}(\lambda)$, of the linearized around the minimal solution $u_{\lambda}$, i.e.

$$
\left\{\begin{array}{l}
L \xi-\lambda q m(x) u_{\lambda}^{q-1} \xi=\gamma_{1}(\lambda) \xi \text { in } \Omega, \\
\frac{\partial \xi}{\partial n}-r a(x) u_{\lambda}^{r-1} \xi=\gamma_{1}(\lambda) \xi \quad \text { on } \partial \Omega
\end{array}\right.
$$

or equivalently, the unique zero of the map

$$
\beta(\sigma)=\sigma_{1}\left[L-\lambda q m(x) u_{\lambda}^{q-1}-\sigma, \mathcal{N}-r a(x) u_{\lambda}^{r-1}-\sigma\right] .
$$

\section{Lemma 5.1 .}

(1) If $u_{\lambda}$ is the minimal solution of (5), then $\gamma_{1}(\lambda) \geq 0$.

(2) If $\gamma_{1}\left(\lambda_{0}\right)>0$, for some $\lambda_{0}>0$, then the set of positive solutions of (5) can be parametrized in a neighborhood of $\left(\lambda_{0}, u_{0}\right)$ by a regular and increasing function on $\lambda$.

(3) If $\gamma_{1}\left(\lambda_{0}\right)=0$, for some $\lambda_{0}>0$, then the set of positive solutions of (5) can be parametrized by a new parameter $s \in(-\varepsilon, \varepsilon)$, such that $(\lambda(s), u(s))$ is a positive solution of (5) and

$$
\lambda(s)=\lambda_{0}+s^{2} \lambda_{2}+o\left(s^{3}\right), \quad u(s)=u_{\lambda_{0}}+s \Phi_{0}+s^{2} \Psi_{0}+o\left(s^{3}\right),
$$

where $\Phi_{0}$ is the positive eigenfunction associated to $\gamma_{1}\left(\lambda_{0}\right)$ and $\int_{\Omega} \Phi_{0} \Psi_{0}=0$. Moreover,

$$
S g\left(\lambda^{\prime}(s)\right)=S g\left(\gamma_{1}(u(s))\right) .
$$

Finally, if $L$ is self-adjoint,

$$
\lambda_{2}<0
$$

where $\lambda_{2}$ is defined in (41). 
Remark 5.3. Except the first paragraph, the result is true for any positive solution $u$ not necessarily being the minimal.

Proof: (1) Assume that $\gamma_{1}<0$ and denote by $\phi_{1}$ the positive eigenfunction associated to $\gamma_{1}$. It is not difficult to show (see Ref. ${ }^{11}$ ) that $u_{\lambda}-\alpha \phi_{1}$ is supersolution of (5), for $\alpha>0$ small. Since $u_{\lambda}>v_{\lambda}$, where $v_{\lambda}$ is the unique positive solution obtained in Case $1(a(x)<0)$, and $v_{\lambda}$ is subsolution of $(5)$, it follows the existence of a solution $u<u_{\lambda}$ of (5), an absurdum because $u_{\lambda}$ is the minimal solution.

(2)-(3) Except (43), these two paragraphs follow by el Propositions 20.6, 20.7 and 20.8 of Ref. ${ }^{1}$. Using (41) and the definition of $\Phi_{0}$, we get

$$
\lambda_{2}=\frac{\int_{\Omega} \lambda_{0} \frac{q(1-q)}{2} m(x) u_{\lambda_{0}}^{q-2} \Phi_{0}^{3}+\int_{\partial \Omega} \frac{r(1-r)}{2} a(x) u_{\lambda_{0}}^{r-2} \Phi_{0}^{3}}{\int_{\Omega} m(x) u_{\lambda_{0}}^{q} \Phi_{0}} .
$$

To determine the sign of $\lambda_{2}$, we use the Picone's identity, see for instance Lemma 4.1 in ${ }^{19}$. Taking $\Psi(t)=t^{2}, v=\Phi_{0}$ and $u=u_{\lambda_{0}}$ we get

$$
\int_{\Omega} \lambda_{0}(1-q) m(x) u_{\lambda_{0}}^{q-2} \Phi_{0}^{3}+\int_{\partial \Omega}(1-r) a(x) u_{\lambda_{0}}^{r-2} \Phi_{0}^{3}<0,
$$

whence it follows that $\lambda_{2}<0$.

As an easy consequence we obtain:

Corollary 5.1. Assume $L$ self-adjoint and let $\left(\lambda_{0}, u_{0}\right)$ be a positive solution of (5) with $\lambda=\lambda_{0}>0$, such that $\gamma_{1}\left(\lambda_{0}\right)=0$ Then, there exists $\varepsilon>0$ such that for each $\lambda \in\left(\lambda_{0}-\varepsilon, \lambda_{0}\right)$, (5) has two positive solutions, one of them $l$. a. $s$ and the other one linearly unstable. Moreover, there exists a neighborhood of $\left(\lambda_{0}, u_{0}\right)$ such that (5) does not have a positive solution for $\lambda>\lambda_{0}$.

Now, we will prove paragraph (3) of Theorem 5.5. Assume that there exists a second solution

$$
w=u_{\lambda}+v
$$

where $v>0$ and $\|w\|_{\infty}<\delta$.

Consider $v_{1}$ the solution of (39). Then, there exists $\beta>0$ such that

$$
0=\sigma_{1}\left[L-m(x) v_{1}^{q-1}, \mathcal{N}\right]<\beta<\sigma_{1}\left[L-q m(x) v_{1}^{q-1}, \mathcal{N}\right] .
$$

We claim that

$$
\sigma_{1}\left[L-q m(x) v_{1}^{q-1}, \mathcal{N}-a_{M} r \delta^{r-1}\right]<0
$$


which is an absurdum with (45). In order to prove (46), it suffices to prove that $v$ is a positive subsolution of $\left(L-q m(x) v_{1}^{q-1}, \mathcal{N}-a_{M} p \delta^{p-1}, \Omega\right)$. Indeed, since $w$ is solution of (5) and by the concavity of the map $t^{q}$, it follows that

$$
L v \leq \lambda m(x) q u_{\lambda}^{q-1} v .
$$

But, since $\lambda^{1 /(1-q)} v_{1}$ is subsolution of (5), then $u_{\lambda}>\lambda^{1 /(1-q)} v_{1}$, and hence

$$
\left(L-q m(x) v_{1}^{q-1}\right) v<0 .
$$

On the other hand, $a(x)\left[\left(u_{\lambda}+v\right)^{r}-u_{\lambda}^{r}\right] \leq a_{M} r \delta^{r-1} v$, whence

$$
\frac{\partial v}{\partial n}-a_{M} r \delta^{r-1} v \leq 0
$$

The following result shows that all the positive solution of (5) are unstable for $\lambda \leq 0$.

Lemma 5.2. If $u$ is a positive solution of (5) for $\lambda \leq 0$, then $u$ is unstable.

Proof: It suffices to prove that

$$
\sigma_{1}\left[L-\lambda q m(x) u^{q-1}, \mathcal{N}-a(x) r u^{r-1}\right]<0 .
$$

First, observe that the first eigenvalue is well defined because $\min _{\bar{\Omega}} u>$ 0 . It remains to find a positive subsolution of $\left(L-\lambda q m(x) u^{q-1}, \mathcal{N}-\right.$ $\left.a(x) r u^{r-1}, \Omega\right)$.

It is hot hard to show that $\underline{u}=u_{\lambda}^{p}$ with $1<p \leq r$ is the desired subsolution.

We are going to finish the proof of the Theorem. Since $r<\frac{N}{N-2}$, by Theorem 4.4, we have that $\mathcal{P}_{\lambda}\left(\mathcal{C}_{0}\right)=(-\infty, \Lambda]$.

We consider the set

$$
\Gamma:=\left\{\left(\lambda, u_{\lambda}\right): \lambda>0, \gamma_{1}(\lambda)>0\right\} .
$$

We claim that $\Lambda=\sup \Gamma$. By Lemma 5.1, the uniqueness of solution with small norm and Corollary 5.1, it follows that $\sup \Gamma=\widetilde{\lambda}>0$. It is clear that $\widetilde{\lambda} \leq \Lambda$. Assume that $\widetilde{\lambda}<\Lambda$, then there exists $\lambda_{0}>\widetilde{\lambda}$ such that $u_{\lambda_{0}}$ is supersolution of (5) for all $\lambda \leq \lambda_{0}$. Since we always can build small subsolutions, then there exists a solution $u^{\lambda}$ for $\lambda<\lambda_{0}$. Since $u^{\lambda}$ is built by the sub-supersolution, then $\gamma_{1}\left(u^{\lambda}\right) \geq 0$, and so, by Lemma 5.1 and Corollary 5.1 we have that there exists $\bar{\lambda}>\tilde{\lambda}$ such that

$$
\gamma_{1}\left(u^{\bar{\lambda}}\right)>0 .
$$


Now, we can continue this solution to the left, we call

$$
\Gamma_{0}:=\left\{\left(\lambda, u^{\lambda}\right): \lambda<\bar{\lambda}\right\}
$$

to this new set. It can occur four possibilities. First, there exists $\lambda_{2} \in(0, \widetilde{\lambda})$ such that $u_{\lambda_{2}}=u^{\lambda_{2}}$, which is not possible because around a l.a.s. solution there is not another solution. Second, there exits $\lambda_{3}$ such that $u^{\lambda_{3}}=0$. Recall that the unique bifurcation point is $\lambda=0$, so this is not possible. Third, there exists $u^{\lambda}$ for all $\lambda \leq \lambda_{0}$, a contradiction with Lemma 5.2. Finally, there exists $\lambda_{4}$ such that $\gamma_{1}\left(u^{\lambda_{4}}\right)=0$, which is impossible by Lemma 5.1 and Corollary 5.1. This proves that $\tilde{\lambda}=\Lambda$.

With a similar reasoning it can be proved the uniqueness of 1 . a. s. positive solution. For the existence of two positive solutions for all $\lambda \in$ $(0, \Lambda)$ it is used the fixed point index respect to the positive cone. Basically, the total index is zero and the index of $u_{\lambda}$ equals one: other positive solution must exist, see Refs. ${ }^{11}$ and ${ }^{17}$.

\section{References}

1. H. Amann, SIAM Rewiew, 18, 620 (1976).

2. H. Amann, Israel J. of Maths. 45, 225 (1983).

3. H. Amann, "New Developments in Diff. Eqns" (Eckhaus, W., ed.), Math. Studies, 21, North-Holland, Amsterdam, 43 (1976).

4. H. Amann and J. López-Gómez, J. Diff. Eqns. 146, 336 (1998).

5. A. Ambrosetti and P. Hess, J. Math. Anal. Appl. 73, 411 (1980).

6. F. Andreu, J. M. Mazón, J. Toledo and J. D. Rossi, Nonl. Anal. 49, 541 (2002).

7. J. M. Arrieta, R. Pardo and A. Rodríguez-Bernal, submitted (2004).

8. S. Cano-Casanova, in this Volume.

9. S. Cano-Casanova and J. López-Gómez, J. Diff Eqns. 178, 123 (2002).

10. M. Chipot, M. Fila and P. Quittner, Acta Math. Univ. Comenianae, 60, 35 (1991).

11. M. Delgado and A. Suárez, Houston J. of Math. 29, 801 (2003).

12. J. Fernández Bonder and J. D. Rossi, Adv. Diff. Eqns. 6, 1 (2001).

13. J. L. Gámez, Nonl. Anal. 28, 625 (1997).

14. J. García Azorero, I. Peral and J. D. Rossi, J. Diff. Eqns., 198, 91 (2004).

15. J. García-Melián, J. D. Rossi and J. C. Sabina de Lis, submitted (2004).

16. D. Gilbarg and N. S. Trudinger, Elliptic partial differential equations of second order, Springer Verlag (1983).

17. R. Gómez-Reñasco and J. López-Gómez, J. Diff. Eqns., 167, 36 (2000).

18. A. W. Leung and Q. Zhang, Math. Meth. Appl. Sci. 21, 1593 (1998).

19. J. López-Gómez, Comm. Partial Differential Equations, 22, 1787 (1997).

20. J. López-Gómez, Adv. Diff. Eqns. 8, 1025 (2003).

21. J. López-Gómez, V. Márquez and N. Wolanaski, Revista Un. Mat. Argentina 38, 196 (1993). 
22. W. Mingxin and W. Yonghui, Chin. Ann. of Math. 16B, 371 (1995).

23. C. Morales-Rodrigo, DEA Memory, University of Sevilla, (2004).

24. C. Morales-Rodrigo and A. Suárez, submitted (2004).

25. C. V. Pao, Nonlinear parabolic and elliptic equations, Plenum, New York, (1992).

26. P. Quittner, Comment. Math. Univ. Carolinae, 34, 105 (1993).

27. K. Umezu, J. Math. Anal. Appl., 267, 651 (2002).

28. K. Umezu, Nonl. Anal. 49, 817 (2002).

29. K. Umezu, Communications in Applied Analysis, 8, 533 (2004).

30. M. Zhu, J. Diff. Eqns., 193, 180 (2003). 